\title{
Determining Estrogens Using Surface-Assisted Laser Desorption/Ionization Mass Spectrometry with Silver Nanoparticles as the Matrix
}

\author{
Tai-Chia Chiu, ${ }^{\mathrm{a}}$ Lin-Chau Chang, ${ }^{\mathrm{b}}$ Cheng-Kang Chiang, ${ }^{\mathrm{c}}$ and \\ Huan-Tsung Chang ${ }^{\mathrm{C}}$ \\ ${ }^{a}$ Department of Nature Science and Education, National Taitung University, Taitung, Taiwan \\ b School of Pharmacy, College of Medicine, National Taiwan University, Taipei, Taiwan \\ c Department of Chemistry, National Taiwan University, Taipei, Taiwan
}

\begin{abstract}
We describe the application of silver nanoparticles (Ag NPs) as matrices for the determination of three estrogens using surface-assisted laser desorption/ionization mass spectrometry (SALDI-MS). Because Ag NPs have extremely high absorption coefficients $\left(1.2 \times 10^{8} \mathrm{M}^{-1} \mathrm{~cm}^{-1}\right)$ at $337 \mathrm{~nm}$, they are effective SALDI matrices when using a nitrogen laser. Three tested estrogens-estrone (E1), estradiol (E2), and estriol (E3)—adsorb weakly onto the surfaces of the Ag NPs, through van der Waals forces. After centrifugation, the concentrated analytes adsorbed on the Ag NPs were subjected directly to SALDI-MS analyses, with the limits of detection for E1, E2, and E3 being 2.23, 0.23 , and $2.11 \mu \mathrm{M}$, respectively. The shot-to-shot and batch-to-batch variations for the three analytes were less than $9 \%$ and $13 \%$, respectively. We validated the practicality of this present approach through the quantitation of $\mathrm{E} 2$ in human urine. Using this approach, we determined the concentration of E2 in a sample of a pregnant woman's urine to be $0.16 \pm 0.05$ $\mu \mathrm{M}(n=10)$. (J Am Soc Mass Spectrom 2008, 19, 1343-1346) (C) 2008 American Society for Mass Spectrometry
\end{abstract}

$\mathrm{E}$ strogens are steroidal hormones. The most potent naturally occurring estrogen is estradiol (E2), which is interconvertible with estrone (E1). These hormones play important roles during the various stages of mammalian development, including prenatal development, growth, and reproduction, and influence sexual behavior, with E2 displaying the highest estrogenic aciivity. The concentrations of E1, E2, and estriol (E3) in body fluids are closely related to the stages of pregnancy [1].

Current techniques for the analysis of endogenous estrogens and their metabolites employ immunoassays [2], high-performance liquid chromatography [3], capillary electrophoresis [4], and mass spectrometry [5]. Of these techniques, only mass spectrometry provides structural information, but with higher cost and poorer sensitivity (pmol) relative to that of the immunoassaybased analyses (fmol) [6].

Recently, we developed surface-assisted laser desorption/ionization mass spectrometry (SALDI-MS) techniques using $\mathrm{Au}$ nanoparticles (NPs), aptamerfunctionalized $\mathrm{Au}$ NPs, and $\mathrm{TiO}_{2}$ NPs for the analyses of aminothiols, adenosine triphosphate, and catechins,

Address reprint requests to Professor H.-T. Chang, Department of Chemistry, National Taiwan University, 1, Section 4, Roosevelt Rd., Taipei 106, Taiwan. E-mail: changht@ntu.edu.tw respectively [7-9]. SALDI-MS using NPs provides several advantages for the analysis of small molecules $(<500 \mathrm{Da})$, including high reproducibility and a simpler sample preparation procedure [10].

We analyzed estrogens by SALDI-MS using Ag NPs as matrices. We carefully evaluated the effects of the $\mathrm{pH}$ of the sample solutions, the concentration of Ag NPs, and the laser fluence on the estrogens sensitivity of the present SALDI-MS system.

\section{Experimental}

\section{Chemicals}

Citric acid, E1, E2, E3, $\beta$-glucuronidase/sulfatase from Helix pomatia (Type $\mathrm{H}-2$ ), silver nitrate, and trisodium citrate were purchased from Sigma (St. Louis, MO). Ammonia solution $(28 \%)$ was purchased from Showa Chemical Industry (Tokyo, Japan). 1-(+)-Ascorbic acid and dichloromethane were purchased from Acros Organics (Geel, Belgium).

\section{Synthesis and Characterization of Ag NPS}

Ag NPs was prepared through sodium citrate mediated reduction of silver nitrate, using a slight modification of a literature procedure [11]. An aqueous silver nitrate 
solution ( $1 \mathrm{mM}, 100 \mathrm{~mL}$ ) was heated to its boiling point and then trisodium citrate solution $(20 \% \mathrm{wt} / \mathrm{vol}, 0.5$ $\mathrm{mL}$ ) was added while stirring. The solution was heated under reflux for an additional $10 \mathrm{~min}$. A double-beam UV-vis spectrometer (Cintra 10e; GBC Scientific Equipment Pty Ltd., Victoria, Australia) was used to measure the absorbance of the as-prepared Ag NPs. The surface plasmon resonance wavelength of the Ag NPs appears at $410 \mathrm{~nm}$. The size of the Ag NPs was further confirmed through analyses of transmission electron microscopy (TEM) images (H7100; Hitachi High-Technologies Corp., Tokyo, Japan). The concentration of the as-prepared Ag NPs was ca. $2.0 \times 10^{15} \mathrm{NPs} / \mathrm{L}(3.3 \mathrm{nM})$ [12]. After being centrifuged at 12,000 rpm (Sigma 3K30 centrifuge; Sigma Laborzentrifugen $\mathrm{GmbH}$, Postfach, Germany) for $10 \mathrm{~min}$, the as-prepared Ag NPs were concentrated to the final concentration of $7.0 \times 10^{16} \mathrm{NPs} / \mathrm{L}$, which was estimated by comparing the absorbance values at 410 $\mathrm{nm}$ of the solutions after and before centrifugation.

\section{Preparation of Samples}

Stock solutions $(10 \mathrm{mM})$ of E1, E2, and E3 were prepared separately in methanol. Ammonium citrate buffers at $\mathrm{pH}$ 4.0-11.0 were prepared by adjusting different amount of aqueous ammonia into $50 \mathrm{mM}$ citric acid solutions to a final volume of $50 \mathrm{~mL}$. Mixtures $(1.0 \mathrm{~mL})$ of ammonium citrate (50 mM, pH 10.0, $10 \mu \mathrm{L}$ ), Ag NPs (1.7 nM, 5-500 $\mu \mathrm{L})$, and an estrogen (1-100 $\mu \mathrm{M}, 10 \mu \mathrm{L})$ were equilibrated at ambient temperature and pressure for $30 \mathrm{~min}$ before being centrifuged at 12,000 rpm for 10 min. For each mixture, ca. $970 \mu \mathrm{L}$ of the supernatant was discarded and the bottom portion (ca. $30 \mu \mathrm{L}$ ) contained the Ag NPs and solution, which was further subjected to vortex to provide a homogeneous mixture. A sample (ca. $1 \mu \mathrm{L}$ ) of each mixture was pipetted onto a stainless-steel 384-well MALDI target plate and dried in air at room temperature before SALDI-MS analysis.
Urine samples (ca. $250 \mathrm{~mL}$ ) were collected from a pregnant woman (36 wk, age 35) in 500-mL bottles containing ascorbic acid $(0.25 \mathrm{~g})$ that was used for preservation and stored at $-20^{\circ} \mathrm{C}$ until required for analysis. Because endogenous estrogens and their metabolites excreted in urine are present in glucuronidated (major) and sulfated (minor) forms [13], a hydrolysis step was conducted before SALDI-MS sample preparation. Hydrolysis and extraction were conducted according to the literature [14]. After extraction, the organic phase was collected, which was then subjected to evaporation under a vacuum pump. Solutions of ammonium citrate $(0.5 \mathrm{mM}, \mathrm{pH} 10.0,987.5 \mu \mathrm{L})$ and Ag NPs (1.7 nM, $12.5 \mu \mathrm{L}$ ) were added to the dried urine samples and equilibrated for $30 \mathrm{~min}$. The samples were then centrifuged at 12,000 rpm for $10 \mathrm{~min}$. For each sample, ca. $970 \mu \mathrm{L}$ of the supernatant was discarded. The bottom portion $(30 \mu \mathrm{L})$ contained the Ag NPs, the analytes, and solution, which was further subjected to vortex to provide a homogeneous mixture. Each solution (ca. $1 \mu \mathrm{L}$ ) was pipetted onto a stainless-steel 384-well MALDI target plate and dried in air at room temperature before SALDI-MS analysis.

\section{Estimation of Estrogens Absorbed on Each Ag NP}

A spectrofluorometer (Cary Eclipse, Varian, Palo Alto, CA) was used to measure the native fluorescence $\left(\lambda_{\mathrm{ex}}\right.$ : $\left.266 \mathrm{~nm} ; \lambda_{\mathrm{em}}: 312 \mathrm{~nm}\right)$ of the estrogens. Mixtures $(1.0 \mathrm{~mL})$ of ammonium citrate (50 mM, pH 10.0, $10 \mu \mathrm{L}), \mathrm{Ag}$ NPs $(1.7 \mathrm{nM}, 12.5 \mu \mathrm{L})$, and each estrogen $(100 \mu \mathrm{M}, 10 \mu \mathrm{L})$ were equilibrated at ambient temperature and pressure for $30 \mathrm{~min}$ before being centrifuged at 12,000 rpm for 10 min. For each mixture, ca. $970 \mu \mathrm{L}$ of the supernatant was collected for fluorescence measurements. By comparing the fluorescence intensity of the each estrogen in the absence and presence of the Ag NPs, the average number of estrogen molecules adsorbed to each Ag NP was estimated [8].

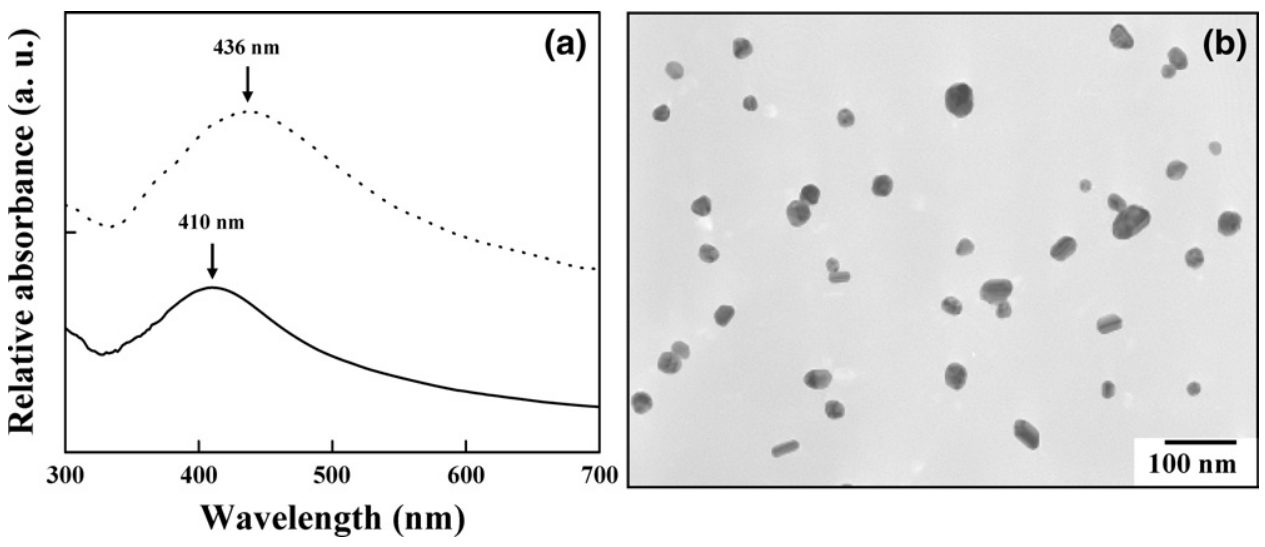

Figure 1. (a) Absorption spectra of Ag NP $(0.02 \mathrm{nM})$ solutions in the absence (solid line) and presence (dotted line) of E2 $(100 \mu \mathrm{M})$. (b) TEM image of Ag NPs. 
Table 1. Impacts of Ag NP concentration, buffer $\mathrm{pH}$, and laser fluence on the $\mathrm{S} / \mathrm{N}$ of estradiol

\begin{tabular}{|c|c|c|c|c|c|}
\hline \multirow[b]{2}{*}{ Ag NPs concentration $(\mathrm{pM})^{a}$} & \multicolumn{5}{|c|}{$\mathrm{S} / \mathrm{N}$ ratios of E2 peak } \\
\hline & 84.5 & 42.2 & 22.1 & 8.5 & 4.2 \\
\hline & $136(33 \%)^{b}$ & $155(25 \%)$ & $130(11 \%)$ & $22(11 \%)$ & $10(7 \%)$ \\
\hline \multirow[t]{2}{*}{ Buffer $\mathrm{pH}^{\mathrm{c}}$} & 4.0 & 6.0 & 8.0 & 10.0 & 11.0 \\
\hline & $21(15 \%)$ & $8(8 \%)$ & $66(28 \%)$ & $130(11 \%)$ & $90(31 \%)$ \\
\hline \multirow[t]{2}{*}{ Laser fluence $(\mu \mathrm{J})^{\mathrm{d}}$} & 102.0 & 102.9 & 103.8 & 104.7 & 105.6 \\
\hline & $25(27 \%)$ & $130(11 \%)$ & $145(17 \%)$ & $103(24 \%)$ & $21(32 \%)$ \\
\hline
\end{tabular}

${ }^{\mathrm{a}} \mathrm{pH}=10.0$ and laser fluence $=102.9 \mu \mathrm{J}$.

b RSD, $n=10$.

${ }^{c}[$ Ag NPs $]=0.02 \mathrm{nM}$ and laser fluence $=102.9 \mu \mathrm{J}$.

${ }^{d}[$ Ag NPs $]=0.02 \mathrm{nM}$ and $\mathrm{pH}=10.0$.

\section{SALDI-MS Measurement}

MS experiments were performed in the negative-ion mode on a reflectron-type time-of-flight (TOF) mass spectrometer (Biflex III; Bruker Daltonics, Bremen, Germany) equipped with a $1.25 \mathrm{~m}$ flight tube [10]. To obtain good resolution and signal-to-noise $(\mathrm{S} / \mathrm{N})$ ratios, the laser fluence was adjusted to slightly higher than the threshold $(102 \mu \mathrm{J})$, and each mass spectrum was generated by averaging 250 laser shots.

\section{Results and Discussion}

\section{Adsorption of Estrogens on $\mathrm{Ag} N \mathrm{NS}$}

Figure 1a and $\mathrm{b}$ displays the UV-vis spectra (solid curve) and TEM image of the Ag NPs solutions, with a maximum absorption wavelength at $410 \mathrm{~nm}$. From counting of $300 \mathrm{Ag} \mathrm{NPs}$, we calculated the average size to be $34( \pm 3) \mathrm{nm}$. In the presence of E2 $(100 \mu \mathrm{M})$, the SPR band shifted to $436 \mathrm{~nm}$ and the full width at half magnitude was broadened from 94 to $128 \mathrm{~nm}$, suggesting their adsorption onto the surfaces of the Ag NPs. We note that the adsorption of the estrogens (neutral) onto the Ag NPs (hydrophobic surface) occurred through van der Waals interactions. After centrifugation, the mixture volume was reduced from $1.0 \mathrm{~mL}$ to 30

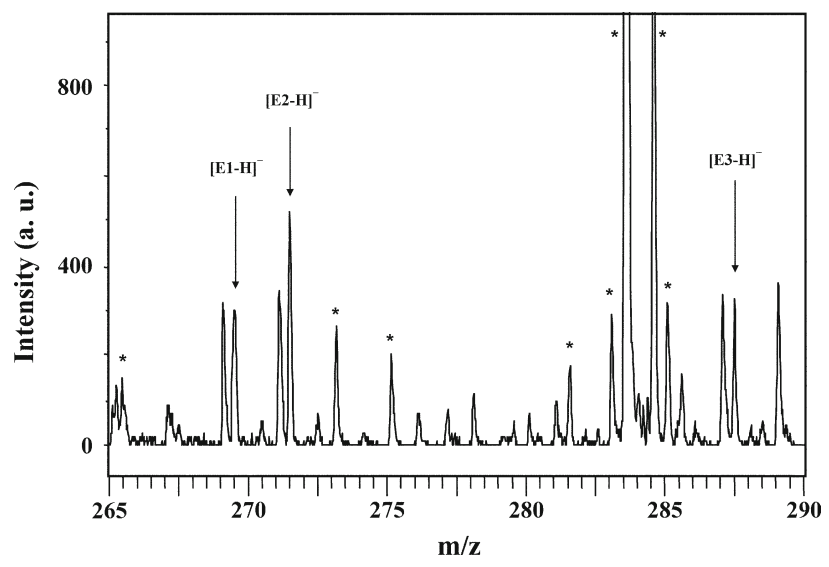

Figure 2. SALDI mass spectrum of a mixture of E1 $(100 \mu \mathrm{M})$, E2 $(10 \mu \mathrm{M})$, and E3 $(100 \mu \mathrm{M})$. Peaks from the background are marked as asterisks. $\mu \mathrm{L}$, leading to concentration of estrogens on the $\mathrm{Ag}$ NPs.

\section{Optimization of SALDI-MS Analysis}

We found that a high mass signal background noise was usually generated at high NP concentrations. From fluorescence data, we estimated that there were $4.5 \times$ $10^{4}$ molecules of E2 on each Ag NP, which corresponds to a saturation concentration of $\mathrm{E} 2$ of $0.90 \mu \mathrm{M}$ when using $0.02 \mathrm{nM}\left(1.2 \times 10^{13} \mathrm{Ag} \mathrm{NPs} / \mathrm{L}\right)$.

Table 1 shows that the optimal conditions for the concentration of $\mathrm{Ag} \mathrm{NPs}, \mathrm{pH}$ of ammonium citrate buffer, and laser fluence were $0.02 \mathrm{nM}, 10.0$, and 102.9 $\mu \mathrm{J}$, respectively. The signals and $\mathrm{S} / \mathrm{N}$ ratios at $\mathrm{pH} 10.0$ were greater than those at $\mathrm{pH} 4.0$ and 6.0. Because the $\mathrm{p} K_{\mathrm{a}}$ of $\mathrm{E} 2$ is 10.2 [16], ca. $0.0006 \%, 0.006 \%$, and $59 \%$ of E2 exist in the deprotonated form at $\mathrm{pH} 4.0,6.0$, and 10.0, respectively. Similarly, the degrees of dissociation of E1 $\left(\mathrm{p} K_{\mathrm{a}} 10.4\right)$ and $\mathrm{E} 3\left(\mathrm{p} K_{\mathrm{a}} 10.0\right)$ both increase upon increasing $\mathrm{pH}[15,16]$. The low $\mathrm{S} / \mathrm{N}$ ratios obtained from the acidic buffers were due to the low desorption/ionization efficiency of E2 when the SALDI-MS system was operated in the negative ion mode.

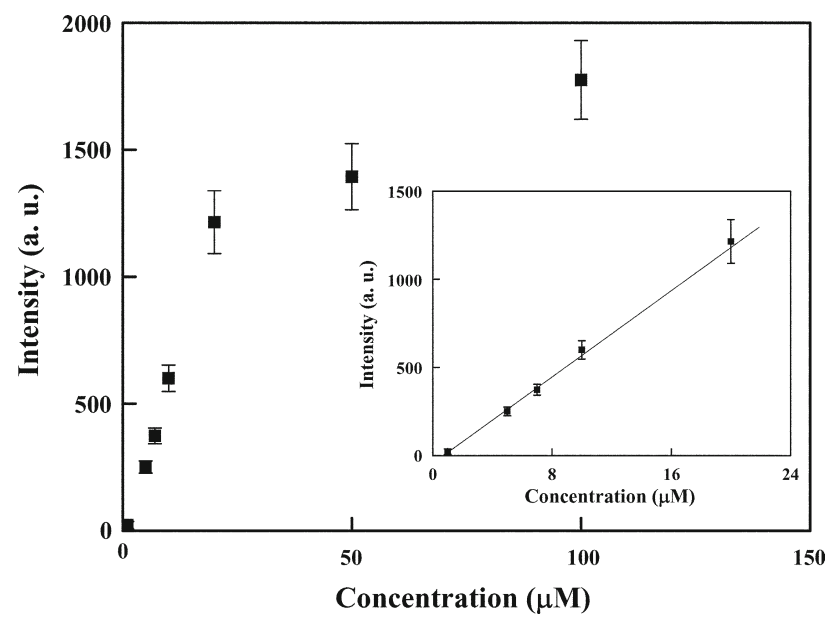

Figure 3. MS signal intensity of the $[\mathrm{E} 2-\mathrm{H}]^{-}$ion plotted against the concentration of E2 $(1-100 \mu \mathrm{M})$. The inset displays the linearity of the plot of the signal intensity for $\mathrm{E} 2$ against its concentration over the range from 1 to $20 \mu \mathrm{M}$. 


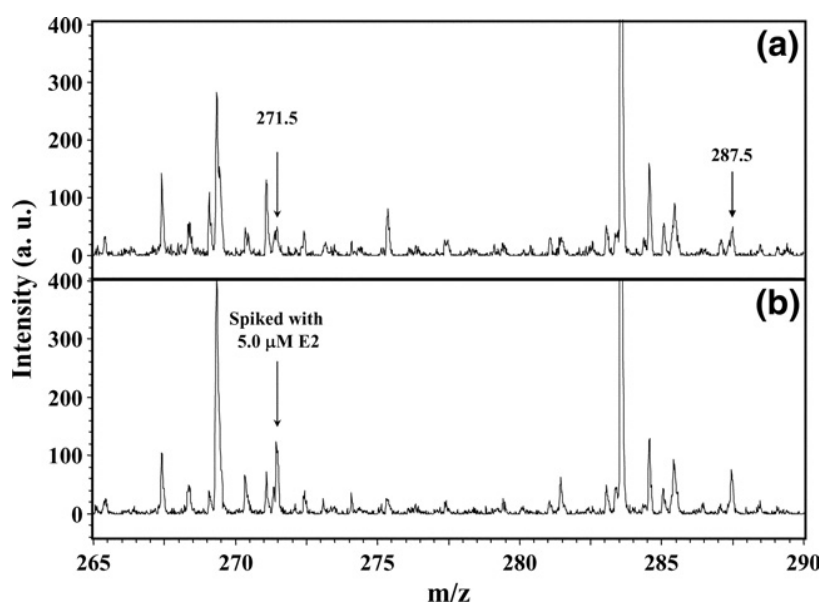

Figure 4. SALDI mass spectra of a pregnant woman's urine: (a) original sample and (b) sample spiked with $5.0 \mu \mathrm{M}$ E2.

Under optimum conditions, we detected the major ion peaks for E1, E2, and E3 at $m / z$ 269.6, 271.5, and 287.5, respectively, which we assigned to the ions [E1 $\mathrm{H}]^{-},[\mathrm{E} 2-\mathrm{H}]^{-}$, and $[\mathrm{E} 3-\mathrm{H}]^{-}$, respectively. The mass spectrum presented in Figure 2 displays the simultaneous analysis of E1, E2, and E3. Each of the three mass spectra displays two high-intensity peaks at $m / z 283.6$ and 284.6, which we assign to [citrate $\left.+2 \mathrm{~K}+\mathrm{NH}_{3}\right]^{-}$ and its deuterated congener, respectively. On the basis of the peak heights, we estimated the LOD at values of $\mathrm{S} / \mathrm{N}$ of 3 for E1, E2, and E3 to be 2.23, 0.23, and $2.11 \mu \mathrm{M}$, respectively. We note that SALDI-MS using Ag NPs provides advantages of small shot-to-shot (all less than $9 \%, n=10$ ), batch-to-batch (all less than 13\%, $n=7$ ) variations.

\section{Determination of E2 in Human Urine}

We obtained a calibration curve of the MS signals for E2 against its concentrations in the range from 1 to $100 \mu \mathrm{M}$ (Figure 3). The inset in Figure 3 indicates that good linearity $\left(R^{2}=0.998\right)$ existed in the range from 1 to 20 $\mu \mathrm{M}$. Next, we tested the practicality of this present SALDI-MS approach through the determination of E2 in a pregnant woman's urine. Figure 4 displays a representative mass spectrum of the urine samples. The signals for the ions of E2 $(m / z 271.5)$ and E3 $(m / z 287.5)$ are readily identifiable. By comparing the peak high with the standard calibration curve, we estimated that the concentrations of E2 and E3 in the pregnant woman's urine were $0.16( \pm 0.05)$ and $0.83( \pm 0.13) \mu \mathrm{M}(n=$ $10)$, respectively, which is in good agreement with literature values $[4,17]$.

\section{Conclusions}

In this study, we employed Ag NPs as SALDI-MS matrices for the analysis of estrogens. When using Ag NPs as matrices, the LOD of E2 was as low as $0.23 \mu \mathrm{M}$.
We used this approach, with its advantages of simplicity, repeatability, and sensitivity, to determine the level of E2 in a pregnant woman's urine. One drawback of using Ag NPs for the determination of estrogens, however, is that their interactions are weak and nonspecific, leading to an inefficient concentration effect. To further improve the sensitivity of the SALDI-MS approach when determining estrogens in a urine sample, we will focus on the preparation of Ag NPs bioconjugated with antibodies against estrogens in future studies.

\section{Acknowledgments}

The authors acknowledge support of this study by the National Science Council of Taiwan under contract no. NSC 95-2113-M-002026-MY3 and NSC 96-2627-M-002-014.

\section{References}

1. Auranen, A.; Hietanen, S.; Salmi, T.; Grenman, S. Hormonal Treatments and Epithelial Ovarian Cancer Risk. Int. J. Gynecol. Cancer 2005, 15, 692-700.

2. Spierto, F. W.; Gardner, F.; Smith, S. J. Evaluation of an EIA Method for Measuring Serum Levels of the Estrogen Metabolite 2-Hydroxyestrone in Adults. Steroids 2001, 66, 59-62.

3. Almeida, C.; Nogueira, J. M. F. Determination of Steroid Sex Hormones in Water and Urine Matrices by Stir Bar Sorptive Extraction and Liquid Chromatography with Diode Array Detection. J. Pharm. Biomed. Anal. 2006, 41, 1303-1311.

4. Su, P.; Zhang, X.-X.; Chang, W.-B. Development and Application of a Multi-Target Immunoaffinity Column for the Selective Extraction of Natural Estrogens from Pregnant Women's Urine Samples by Capillary Electrophoresis. J. Chromatogr. B 2005, 816, 7-14.

5. Xu, X.; Roman, J. M.; Veenstra, T. D.; Van Anda, J.; Ziegler, R. G.; Issaq, H. J. Analysis of Fifteen Estrogen Metabolites Using Packed Column Supercritical Fluid Chromatography-Mass Spectrometry. Anal. Chem. 2006, 78, 1553-1558.

6. Szécsi, M.; Tóth, I.; Gardi, J.; Nyári, T.; Julesz, J. HPLC-RIA Analysis of Steroid Hormone Profile in a Virilizing Stromal Tumor of the Ovary. J. Biochem. Biophys. Methods 2004, 61, 47-56.

7. Huang, Y.-F.; Chang, H.-T. Nile Red-Adsorbed Gold Nanoparticle Matrixes for Determining Aminothiols Through Surface-Assisted Laser Desorption/Ionization Mass Spectrometry. Anal. Chem. 2006, 78, 14851493.

8. Huang, Y.-F.; Chang, H.-T. Analysis of Adenosine Triphosphate and Glutathione through Gold Nanoparticles Assisted Laser Desorption/ Ionization Mass Spectrometry. Anal. Chem. 2007, 79, 4852-4859.

9. Lee, K.-H.; Chiang, C.-K.; Lin, Z.-H.; Chang, H.-T. Determining Enediol Compounds in Tea Using Surface-Assisted Laser Desorption/Ionization Mass Spectrometry with Titanium Dioxide Nanoparticle Matrices. Rapid Commun. Mass Spectrom. 2007, 21, 2023-2030.

10. Chiu, T.-C.; Huang, L.-S.; Lin, P.-C.; Chen, Y.-C.; Chen, Y.-J.; Lin, C.-C. Chang, H.-T. Nanomaterial Based Affinity Matrix-Assisted Laser Desorption/Ionization Mass Spectrometry for Biomolecules and Pathogenic Bacteria. Recent Patents Nanotechnol. 2007, 1, 99-111.

11. Lee, P. C.; Meisel, D. Adsorption and Surface-Enhanced Raman of Dyes on Silver and Gold Sols. I. Phys. Chem. 1982, 86, 3391-3395.

12. Huang, M.-F.; Kuo, Y.-C.; Huang, C.-C.; Chang, H.-T. Separation of Long Double-Stranded DNA by Nanoparticle-Filled Capillary Electrophoresis. Anal. Chem. 2004, 76, 192-196.

13. Adlercreutz, H.; Kiuru, P.; Rasku, S.; Wähälä, K.; Fotsis, T. An Isotope Dilution Gas Chromatographic-Mass Spectrometric Method for the Simultaneous Assay of Estrogens and Phytoestrogens in Urine. J. Steroid Biochem. Mol. Biol. 2004, 92, 399-411.

14. Xu, X.; Veenstra, T. D.; Fox, S. D.; Roman, J. M.; Issaq, H. J.; Falk, R.; Saavedra, J. E.; Keefer, L. K.; Ziegler, R. G. Measuring Fifteen Endogenous Estrogens Simultaneously in Human Urine by High-Performance Liquid Chromatography-Mass Spectrometry. Anal. Chem. 2005, 77, Liquid Chrom

15. Nghiem, L. D.; Schafer, A. I.; Waite, T. D. Adsorptive Interactions Between Membranes and Trace Contaminants. Desalination 2002, 147, 269-274.

16. Yamamoto, H.; Liljestrand, H. M.; Shimizu, Y.; Morita, M. Effects of Physical-Chemical Characteristics on the Sorption of Selected Endocrine Disruptors by Dissolved Organic Matter Surrogates. Environ. Sci. Technol. 2003, 37, 2646-2657.

17. Marrian, G. F. The Urinary Estrogens and Their Quantitative Determination. Cancer 1957, 10, 704-706. 